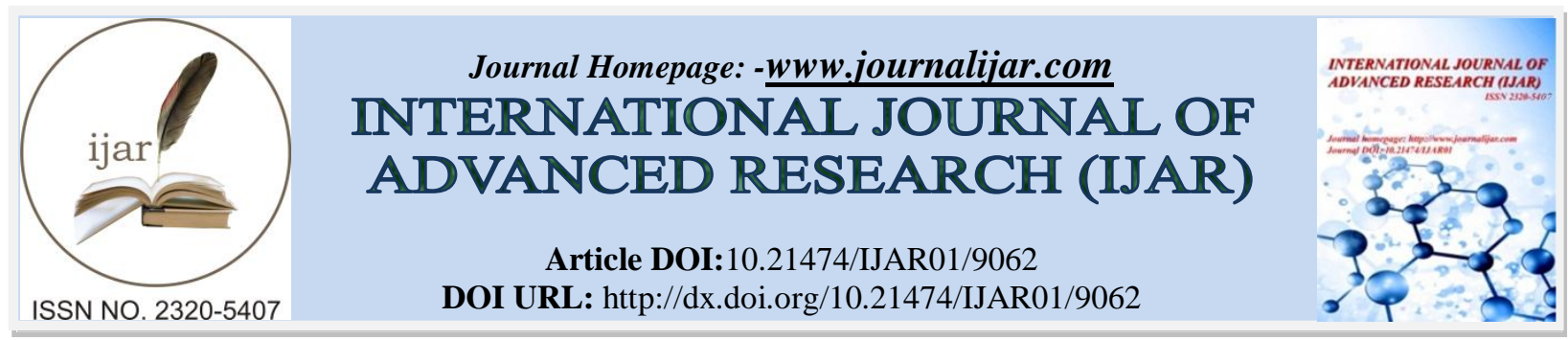

RESEARCH ARTICLE

\title{
DRAG ON AN MICROPOLAR FLOW PAST A SPHERE SPECIFYING UNIFORM VELOCITY AWAY FROM THE BOUNDARIES.
}

Gopinatha K. $\mathbf{R}^{1}$, Jayalakshmamma D. $\mathbf{V}^{2}$ and D. V. Chandrashekhar ${ }^{3}$.

1. Department of Mathematics, Global Academy of Technology, Bangalore-560098.

2. Department of Mathematics, Vemana Institute of Technology, Bangalore, India.

3. Department of Mathematics Vivekananda Institute of Technology, Bangalore, India.

\section{Manuscript Info}

\section{Manuscript History}

Received: 13 March 2019

Final Accepted: 15 April 2019

Published: May 2019

Key words:-

micropolar flow, coupling parameter,

Drag, Uniform shear.

\section{Abstract}

A study of the effect of drag force on anmicropolar flow past a sphere specifying uniform velocity away from the boundaries. We find a similarity solution, assuming the fluid outside the sphere and satisfies the Eringen's micro polar equations and applying no slip condition at the sphere of the surface. An appearance for drag force is obtained. It is found that the increase in the coupling parameter with fixed coupling stress parameter is to decreases drag. Further a reversed behavior is noticed that the drag is increases andthe same is represented graphically.

Copy Right, IJAR, 2019,. All rights reserved.

\section{Introduction:-}

The earliest formulation of a general theory of microcontinua is accredited to Eringen [1] has considered as fluids with deformable microelements. Eringen's [2] 'micropolar fluid theory' is based on the assumptions that the deformation of the fluid microelements is very small. This theory is still capable of taking into account the effect of microrotational surface and body couples. The evaluation of uniform flow past a spherical shell in Newtonian stokes flow has been extensively investigated in the literature, because of its application in lubrication theory, transpiration cooling and other important applications. The stokes uniform flow past a porous sphere has also been investigated by several authors with the assumsion of axisymmtric flow ( Padmavati et al [3], Berman [4], Rudraiah et al [5]). However in environmental pollution problems, particularly in water pollution problem, it is central to consider the effects of suspended particles on the flow past a sphere. The effect of these suspended particles may be taken into account either using Eringen'smicropolar fluid model or using Saffman dusty fluid model. The Saffman dusty fluid model does not much importance of the effect of micro rotation of balanced particles unless we consider principal of angular momentum in addition to linear momentum. The micropolar fluid model has built in mechanism of taking care of micro rotation.

The recently K. Ramalashmi and PankajShukla[6] investigated Drag on a porous sphere embedded in micropolar fluid.Jize Sui, et al [7] is investigation for the shear flow and heat transfer of a micropolar fluid by means of novel constitutive models is valuable. Jian-Jun Shu and JennShiun Lee [8] they obtain fundamental Stokes and Oseen solutions for micropolar flow in three dimensions, so that the point force and point couple can be prescribed in any direction. Karl-Heinz Hoffmann et al [9] they studied the resistant force exerted on a sphere moving with a constant velocity in a micro-polar fluid. 
Presently the analytical study of micropolar fluid flow past an impermeable sphere specifying identical Velocity far From the Boundaries. The expression for drag force is determined. The evaluation of drag coefficient on non dimensional coupling parameter $\mathrm{N}_{1}$ and coupling stress parameter $\mathrm{N}_{3}$ is discussed and presented graphically.

\section{Mathematical Formulation}

Consider a steady incompressible micropolar fluid flow past an impervious sphere of radius ' $a$ ' embedded in a sparsely packed porous medium. The schematic representation is show in diagram under assumptions and approximations made together with governing by the equations of continuity, conservation of momentum and Conservation of angular momentum

$$
\begin{aligned}
& \nabla \cdot \vec{q}=0 . \\
& -\nabla p+\varsigma(\nabla \times \vec{\omega})+(\varsigma+\eta) \nabla^{2} \vec{q}=0 . \\
& \varsigma(\nabla \times \vec{q})-2 \varsigma \rho+\left(\lambda^{\prime}+\eta^{\prime}\right) \nabla\left(\nabla \cdot \rho^{\rho}\right)+\eta^{\prime} \nabla^{2} \vec{\omega}=0 .
\end{aligned}
$$

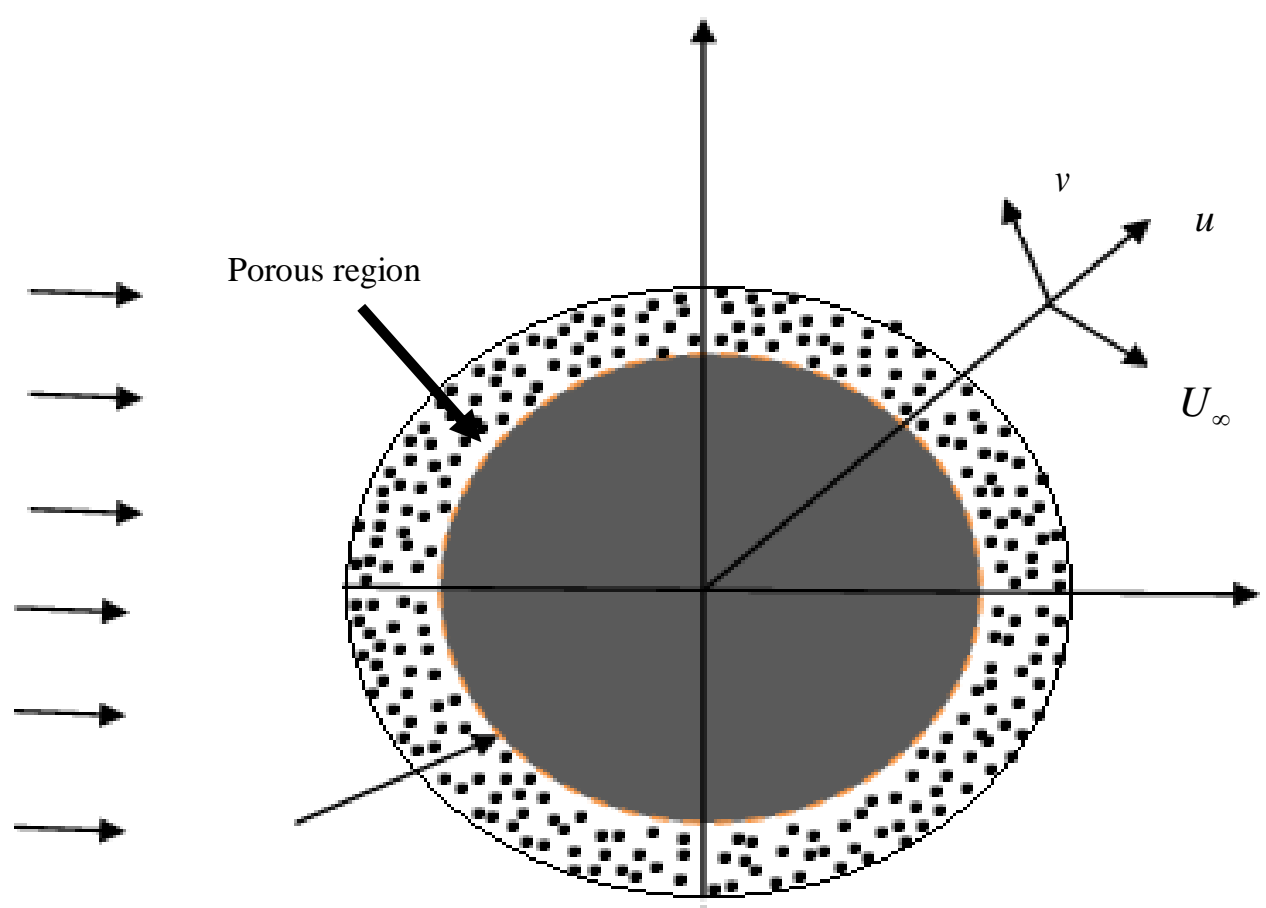

Solid spherical core

$$
H_{0}
$$

\section{Physical configuration}

where $\vec{q}$ is the velocity, $\varsigma$ is the coupling viscosity, $\tilde{\omega}$ is the angular velocity, $p$ is the pressure, $\varsigma$ is the coupling viscosity, $\eta$ is the bulk viscosity co-efficients and $\lambda$ is the shear viscosity co-efficients $\lambda^{\prime}$ is the bulk spin viscosity co-efficients and $\eta^{\prime}$ is the shear spin viscosity co-efficients. 
Stream function $\psi(r, \theta)$ is introduced, such that the equation of continuity is satisfied in spherical polar co-ordinate system for porous regions and defined as:

$u=\frac{1}{r^{2} \sin \theta} \frac{\partial \psi}{\partial \theta} ; \quad v=\frac{-1}{r \sin \theta} \frac{\partial \psi}{\partial r}$.

By removing the pressure term from equations (2) and (3) will obtained as:

$\left(E^{4}-N^{2} E^{2}\right) E^{2} \psi=0$.

Where $E^{2}=\frac{\partial^{2}}{\partial r^{2}}+\frac{\sin \theta}{r^{2}} \frac{\partial}{\partial \theta}\left(\frac{1}{\sin \theta} \frac{\partial}{\partial \theta}\right)$ is the Laplacian operator in spherical co-ordinate system.

$N^{2}=\frac{\left(2-N_{1}\right)}{N_{3}} N_{1} \quad N_{1}=\frac{\varsigma}{\varsigma+\eta} \quad$ Coupling parameter $\quad 0 \leq N_{1} \leq 1$

$N_{3}=\frac{\eta^{\prime}}{(\varsigma+\eta) a^{2}} \quad$ Coupling stress parameter $\quad 0 \leq N_{3} \leq m \quad$ (m: positive real).

We now assume $\psi=\psi_{0}+\psi_{1}$, equation (5) using this resolution we get

$E^{2} \psi_{0}=0$

$E^{4} \psi_{1}-N^{2} E^{2} \psi_{1}=0$

\section{Boundary conditions}

To solve the above governing equation we considered the boundary conditions are no-slip condition given by

$$
\frac{\partial}{\partial \theta} \psi(r, \theta)=\frac{\partial}{\partial r} \psi(r, \theta)=0 \quad \text { at } \quad r=1
$$

The flow has uniform velocity when Far away from the sphere given by

$\psi(r, \theta) \sim \frac{r^{2}}{2} \sin ^{2} \theta$, as $r \rightarrow \infty$.

\section{Method of solution}

The boundary condition from equation (9) suggests the following similarity solution

$\psi(r, \theta)=f(r) \sin ^{2} \theta$.

Substituting equation (10) in (7), then functions $\psi(r, \theta)$ reduces to fourth order ordinary differential equation in $f(r)$ as follows:

$$
f^{i v}(r)-\frac{4}{r^{2}} f^{\prime \prime}(r)+\frac{8}{r^{3}} f^{\prime}(r)-\frac{8}{r^{4}} f(r)-N^{2}\left(f^{\prime \prime}(r)-\frac{2}{r^{2}} f(r)\right)=0 \text {. }
$$

The corresponding $f(r)$, from equation (8) and (9) reduces to: No-slip condition of solid sphere at the surface is given by

$f(1)=0, \quad f^{\prime}(1)=0$. at $\mathrm{r}=1$

Further, the uniform velocity extreme away from the boundary, from equation (10) reduces to: 
$f(r) \sim \frac{r^{2}}{2}$ as $r \rightarrow \infty$

The solution for the equations (11) is obtained analytically by using thetransformation

$$
g(r)=f^{\prime \prime}(r)-\frac{2}{r^{2}} f(r)
$$

Substituting equation (14) in equation (11), it reduces to second order ordinary differential equation in $g(r)$ as,

$$
g^{\prime \prime}(r)-\left(N^{2}+\frac{2}{r^{2}}\right) g(r)=0 \text {. }
$$

Further, consider the transformation function $g(r)$ as

$$
g(r)=\sqrt{r} w(r) \text {. }
$$

Where $w(r)$ the arbitrary function. Thereby, equation (15) reduces to:

$$
r^{2} w^{\prime \prime}(r)+r w^{\prime}(r)-\left[\left(\frac{3}{2}\right)^{2}+(r N)^{2}\right] w(r)=0 \text {. }
$$

Which is the modified Bessel's differential equation, and its solution in terms of modified Bessel's function is given as:

$$
w(r)=C_{1} I_{3 / 2}(r N)+D_{1} K_{3 / 2}(r N)
$$

Where, $C_{1}$ and $D_{1}$ are arbitrary constants. Thus, from equation (18) we have:

$$
g(r)=C_{1} \sqrt{r} I_{3 / 2}(r N)+D_{1} \sqrt{r} K_{3 / 2}(r N) \text {. }
$$

Further, equation (14) reduces to a second order ordinary differential equation with variable co-efficient as:

$$
f^{\prime \prime}(r)-\frac{2}{r^{2}} f(r)=C_{1} \sqrt{r} I_{3 / 2}(r N)+D_{1} \sqrt{r} K_{3 / 2}(r N) \text {. }
$$

Equation (20) is an ordinary differential equation of order two with variable co-efficient; its general solution can be obtained by the method of variation of parameters and is given by:

$$
f(r)=\frac{A_{1}}{r}+B_{1} r^{2}+C_{1} \sqrt{r N} I_{3 / 2}(r N)+D_{1} \sqrt{r N} K_{3 / 2}(r N)
$$

Where $A_{1}$ and $B_{1}$ are arbitrary constants.

as $r \rightarrow \infty$ then $I_{3 / 2}(r N) \rightarrow \infty$. Therefore, the abovesolution is valid if and only if $C_{1}=0$. Hence equation (21) reduces to:

$$
f(r)=\frac{A_{1}}{r}+B_{1} r^{2}+D_{1} \sqrt{r N} K_{3 / 2}(r N) \text {. }
$$

Where $A_{1}, B_{1}, D_{1}$ are constants to be evaluate using the boundary conditions

(8) and (9). Determining these constants and on substitution, equation (22) reduces to:

$$
f(r)=\frac{r^{2}}{2}-\frac{N^{2}+3 N+3}{N^{2}} \frac{1}{2 r}+\frac{3}{2 N}\left(1+\frac{1}{r N}\right) e^{-(r-1) N}
$$

In terms of stream function, from the equation (10) we get: 
$\psi(r, \theta)=\left(\frac{r^{2}}{2}-\frac{N^{2}+3 N+3}{N^{2}} \frac{1}{2 r}+\frac{3}{2 N}\left(1+\frac{1}{r N}\right) e^{-(r-1) N}\right) \sin ^{2} \theta$.

This equations shows the function of coupling parameter and coupling stress parameter

The shearing stress is given by

$\tau_{r \theta}=\bar{\mu}\left(\frac{1}{r} \frac{\partial u}{\partial \theta}+r \frac{\partial}{\partial r}\left(\frac{v}{r}\right)\right)$

On non-dimensionlisingequation (25) reduces to

$$
\frac{\tau_{r \theta}}{\overline{\bar{\mu} U_{\infty}}}=-\left(\frac{1}{r} \frac{\partial u}{\partial \theta}+r \frac{\partial}{\partial r}\left(\frac{v}{r}\right)\right)
$$

When the sphere on the surface (i.e $r=1$ ), the shearing stress becomes

$$
\begin{aligned}
& \frac{\tau_{r \theta}}{\frac{\bar{\mu} U_{\infty}}{a}}=-r \frac{\partial}{\partial r}\left(\frac{v}{r}\right) \\
& \tau_{r \theta}=\frac{\bar{\mu} U_{\infty}}{a} \frac{3}{2}(1+N) \sin \theta .
\end{aligned}
$$

\section{Determination of the Drag force}

The drag force $\mathrm{F}$ experienced by an impermeable sphere of radius ' $\mathrm{a}$ ' is defined by

$$
F_{t}=\int_{0}^{2 \pi} \int_{0}^{\pi}\left\{\left(\tau_{r \theta}\right)_{r=a} \sin \theta\right\} R^{2} \sin \theta d \theta d \phi .
$$

On evaluation, equation (29) reduces to as:

$$
F_{t}=4 \pi\left(\bar{\mu} U_{\infty} a\right)(1+N) .
$$

Further, the drag coefficient can be written as

$$
C_{D}=\frac{-F_{t}}{\frac{1}{2} \rho U_{\infty}^{2} a^{2} \pi}
$$

Substitution of equation (30) in equation (31), itreduces to:

$$
C_{D}=\frac{-16}{R_{e}}(1+N) \text { and } R_{e}=\frac{2 \rho U_{\infty} a}{\bar{\mu}}
$$

Where $R_{e}$ is the Reynolds number and if $S=0$, then equation (30) reduces to:

$$
F_{t}=4 \pi \bar{\mu} U_{\infty} a \text {. }
$$

One of the result of Happel and Brenner [10] 


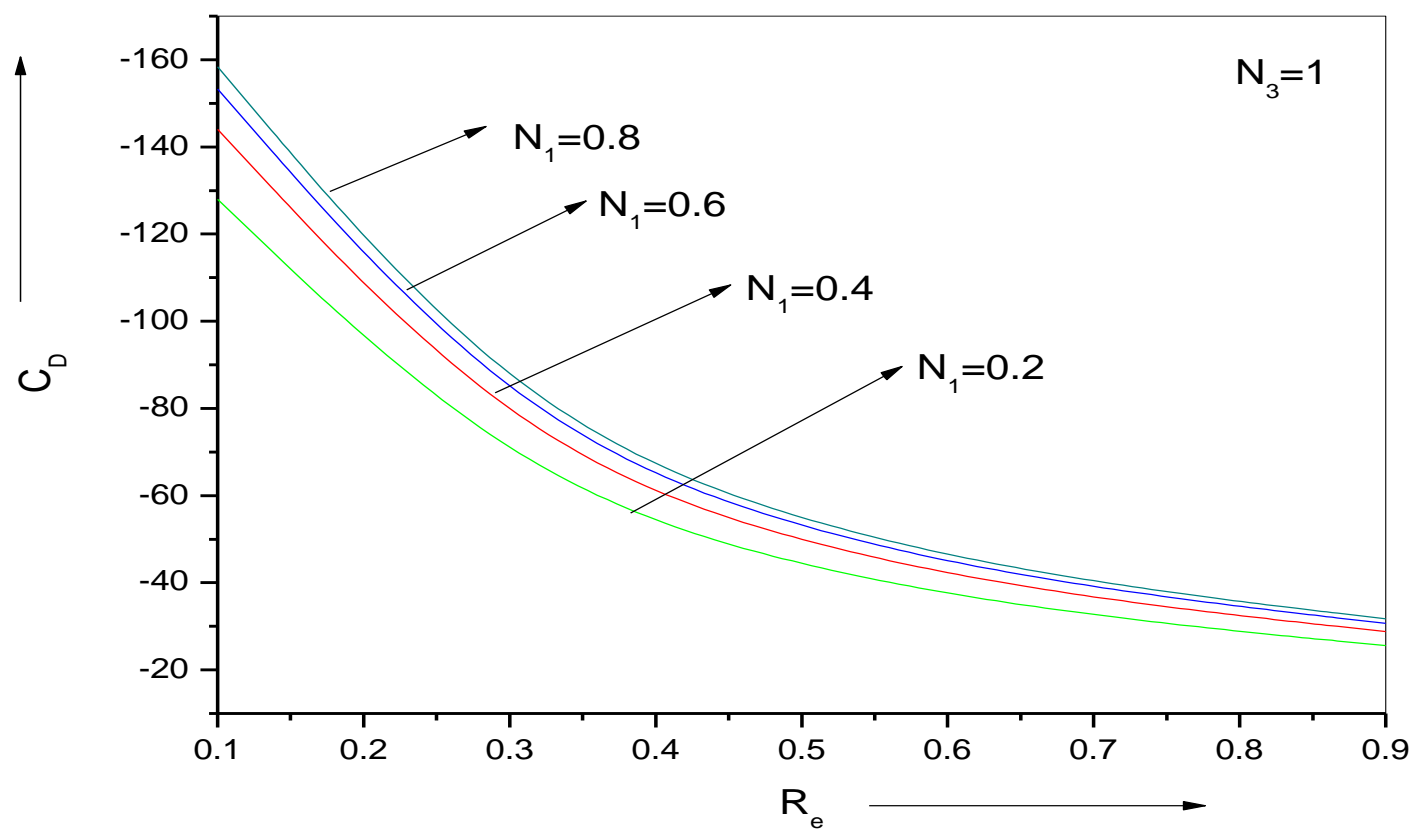

Fig 1:-Dependence of the Drag coefficient on coupling stress parameter $\mathrm{N}_{3}=1$ for various values of coupling parameter $\mathrm{N}_{1}$

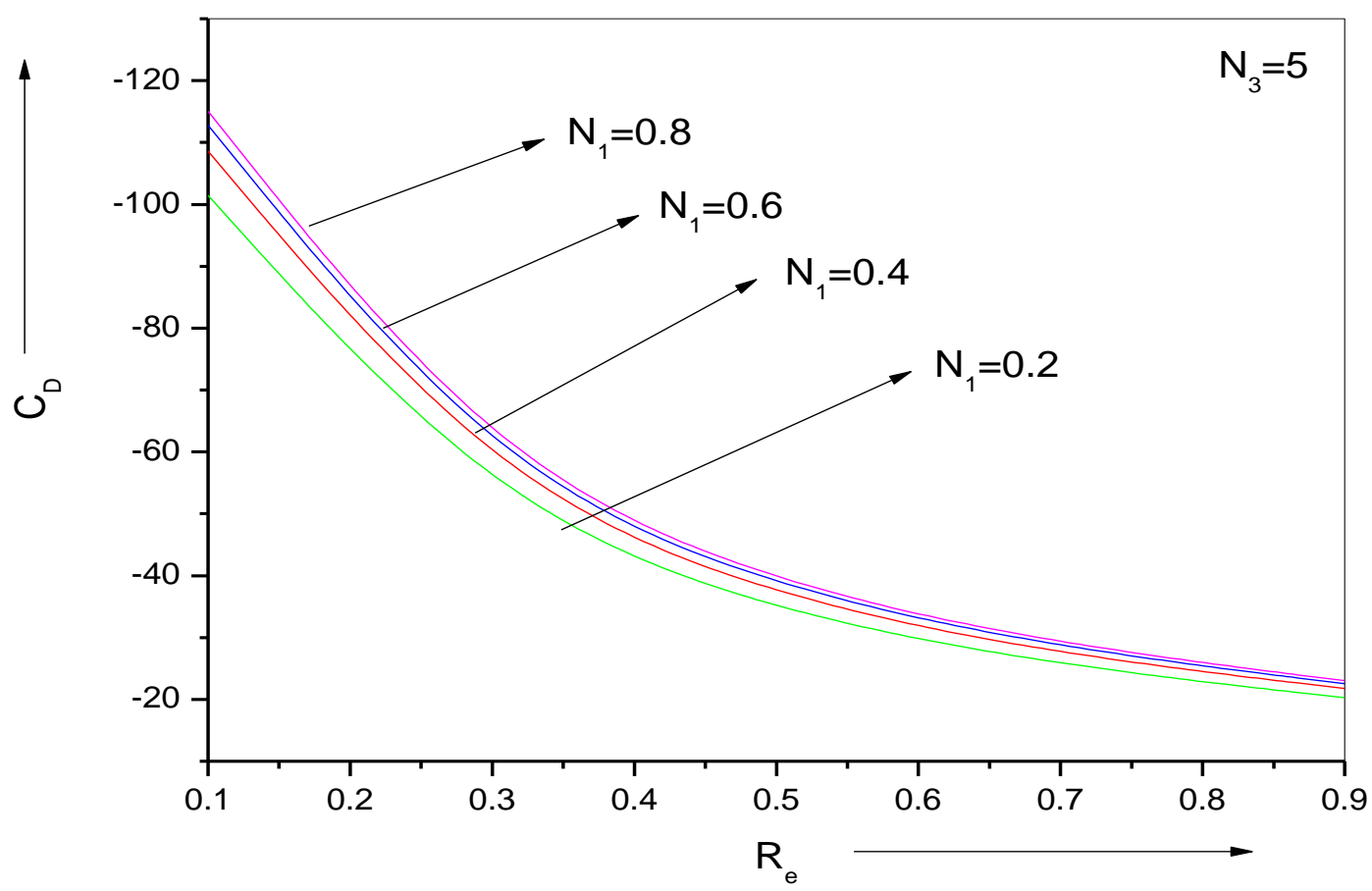

Fig 2:-Dependence of the Drag coefficient on coupling stress parameter $\mathrm{N}_{3}=5$ for various values of coupling parameter $\mathrm{N}_{1}$ 


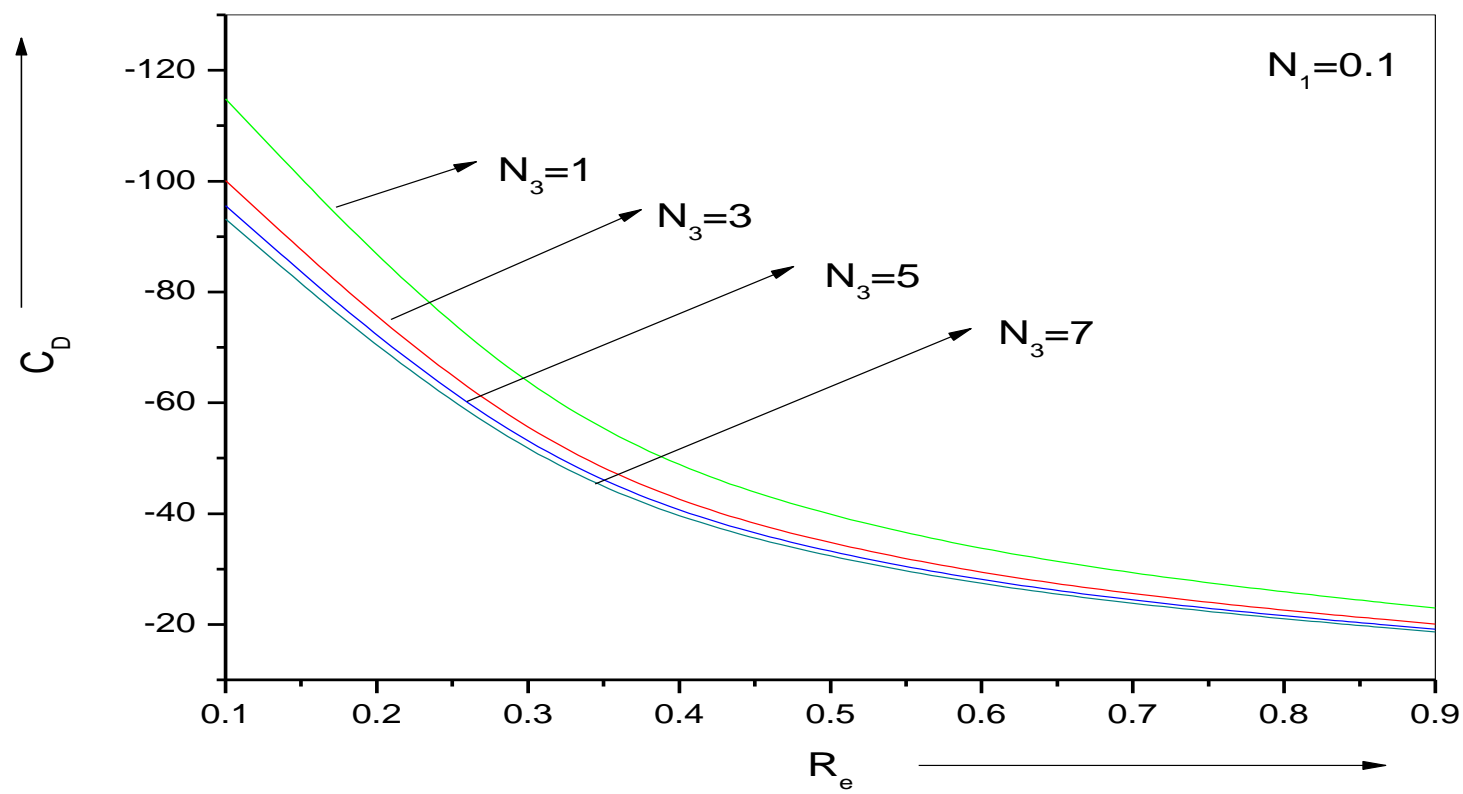

Fig 3:-Dependence of the Drag coefficient on coupling parameter $\mathrm{N}_{1}=0.1$ for various values of coupling stress parameter $\mathrm{N}_{3}$

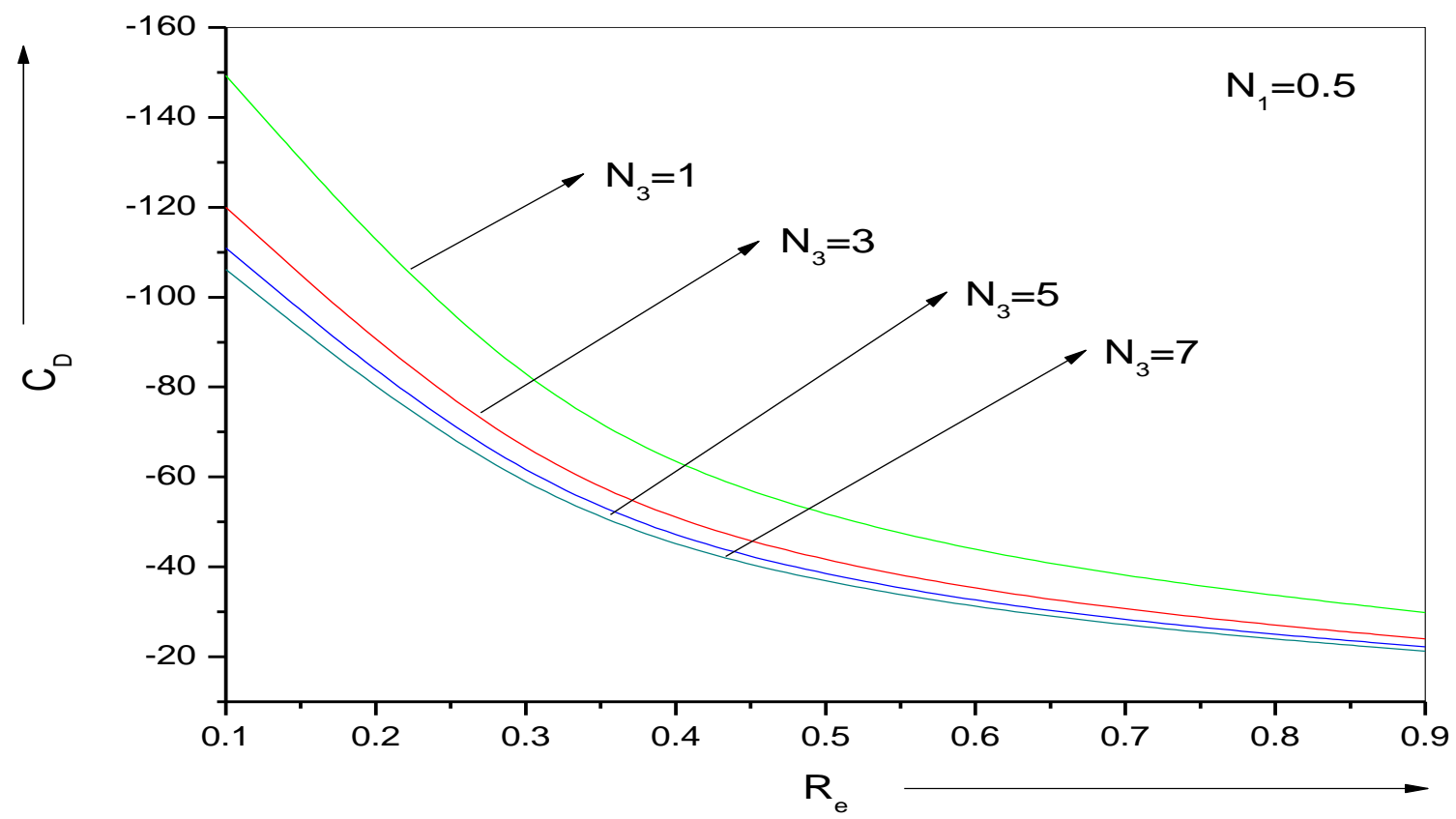

Fig 4:-Dependence of the Drag coefficient on coupling parameter $\mathrm{N}_{1}=0.5$ for various values of coupling stress parameter $\mathrm{N}_{3}$ 


\section{Results:-}

In this paper we study dependence of drag coefficient with variation of coupling parameter and coupling stress parameter for the steady flow of viscous. The drag experienced by a sphere embedded in porous medium, using the no-slip condition at the solid surface and uniform shear flow far away from the region as the boundary conditions. Also, the expression for the drag co-efficient $C_{D}$ is obtained.

From figures 1 and 2, we noticed that the drag co-efficient $C_{D}$ decreases with increase coupling parameter $N_{1}$ for fixed coupling stress parameter $\mathrm{N}_{3}=1$ and $\mathrm{N}_{3}=5$ near the solid surface and maintains asymptotic behavior away from the surface. Further, figures 3 and 4 shows that the increase coupling stress parameter $\mathrm{N}_{3}$, drag co-efficient $\mathrm{C}_{\mathrm{D}}$ is increases for fixed coupling parameter $\mathrm{N}_{1}=0.1$ and $\mathrm{N}_{1}=0.5$.

\section{Acknowledgements:-}

The authors are grateful to research centre Vivekananda Institute of Technology, Vemana Institute of Technology, and Global Academy of Technology, Bangalore, India.

\section{References:-}

1. Eringen, A.C.: Simple microfluids, Int. J. Engng. Sci., 2, 205-217 (1964).

2. Eringen, A.C : Theory of micropolar fluids. J. Math. Mech. 16(1), 1-18. (1966)

3. Padmavathi, B.S., Amaranath, T. and Nigam, S.D., Stokes flow past a porous sphere using Brinkman Model. ZAMP, 1993; 44, 929-939.

4. Pop, I and Ingham, D. B., Flow past a sphere embedded in a porous medium based on the Brinkman model, Int. Commun. Heat mass transfer, vol. 23, pp. 865-874, (1996).

5. Rudraiah, N., Shivakumara, I. S., Palaniappa, D.., and Chandrasekhar, D. V., Flow past an impervious sphere embedded in a porous medium based on non- Darcy model, Proc. Seventh Asian Cong. Fulid Mech., pp. 565$568,(1997)$

6. K. Ramalkshmi, PankajShukla., Drag on a porous sphere embedded in micropolar fluid, Int. J. of Applied mathematics vol 109 No. 10, 181-189(2016)

7. Jize Sui et al "A novel investigation of a micropolar fluid characterized by nonlinearconstitutive diffusion model in boundary layer flow and heat transfer,Physics of fluids 29, 023105 (2017)

8. Jian-Jun Shu\&JennShiun Lee "Fundamental solutions for micropolar fluids" J Eng Math (2008) 61:69-79

9. Karl-Arl--Heinz Hoffmann ,David Marx and Nikolai D. Botkin "Drag on spheres in micropolar fluids with non-zero boundary conditions for microrotations" Article in Journal of Fluid Mechanics · November 2007

10. J. Haappel and H. Brenner: Low Renolds number Hydrodynamics, MartinusNijoff publishers, the Hague, (1983). 